\title{
Atorvastatin increases Fads1, Fads2 and Elovl5 gene expression via the geranylgeranyl pyrophosphate-dependent Rho kinase pathway in 3T3-L1 cells
}

\author{
NORIKO ISHIHARA ${ }^{1}$, SAWAKO SUZUKI ${ }^{2}$, SHOU TANAKA ${ }^{1,2}$, YASUHIRO WATANABE ${ }^{1,2}$, \\ DAIJI NAGAYAMA ${ }^{1}$, ATSUHITO SAIKI ${ }^{1}$, TOMOAKI TANAKA ${ }^{3}$ and ICHIRO TATSUNO ${ }^{1}$ \\ ${ }^{1}$ Center for Diabetes, Metabolism and Endocrinology, Toho University Sakura Medical Center, Sakura, Chiba 285-8741; \\ Departments of ${ }^{2}$ Clinical Cell Biology and ${ }^{3}$ Molecular Diagnosis, Graduate School of Medicine, \\ Chiba University, Chiba, Chiba 260-8670, Japan
}

Received March 28, 2017; Accepted June 21, 2017

DOI: $10.3892 / \mathrm{mmr} .2017 .7141$

\begin{abstract}
Numerous clinical studies have reported that statins increase the plasma concentration of arachidonic acid, which is an $\omega-6$ long-chain polyunsaturated fatty acid (LCPUFA), and decrease the concentrations of eicosapentaenoic acid and docosahexaenoic acid, which are $\omega-3$ LCPUFAs. These findings indicate that statins may affect the endogenous synthesis of LCPUFAs, which is regulated by fatty acid desaturases (FADSs) and elongation of very long-chain fatty acids proteins (ELOVLs). The present study aimed to investigate the roles of the intrinsic mevalonate cascade and Rho-dependent pathway in statin-induced regulation of these desaturases and elongases, as well as cell viability using mouse 3T3-L1 cells. mRNA expression was analyzed by quantitative polymerase chain reaction. Treatment with atorvastatin decreased cell viability and increased the mRNA expression levels of Fads1, Fads2 and ELOVL fatty acid elongase 5 (Elovl5) in a dose-dependent manner. Mevalonate and geranylgeranyl pyrophosphate (GGPP), but not cholesterol, fully reversed the atorvastatin-induced downregulation of cell viability and upregulation of gene expression; however, mevalonate itself did not affect cell viability and gene expression. The Rho-associated protein kinase inhibitor Y-27632 inhibited the mevalonate- and GGPP-mediated reversal of atorvastatin-induced upregulation of Fads1, Fads2 and Elovl5. These findings indicated that statins may affect the endogenous synthesis of LCPUFAs by regulating Fads1, Fads2 and Elovl5
\end{abstract}

Correspondence to: Professor Ichiro Tatsuno, Center for Diabetes, Metabolism and Endocrinology, Toho University Sakura Medical Center, 564-1 Shimoshizu, Sakura, Chiba 285-8741, Japan

E-mail: ichiro.tatsuno@med.toho-u.ac.jp

Key words: atorvastatin, 3T3-L1 cells, fatty acid desaturases, elongation of very long-chain fatty acids, geranylgeranyl pyrophosphate, Rho kinase gene expression via the GGPP-dependent Rho kinase pathway in mouse 3T3-L1 cells.

\section{Introduction}

Arachidonic acid (AA; 20:4 n-6), which is a 20-carbon, 4-double-bond, long-chain polyunsaturated fatty acid (LCPUFA) of the $\omega-6$ type, is an essential fatty acid that is not synthesized by the human body. AA is the major source of eicosanoids, which are lipid modulators of vascular function. Eicosanoids mainly act locally by signaling via specific receptors, and are able to modulate numerous functions, including vasomotor tone, hemostasis, inflammation and cell proliferation. In the pathological setting of atherosclerosis, eicosanoids serve important roles, since they contribute to endothelial dysfunction and induce inflammation, arterial smooth muscle cell hyperplasia and thrombosis (1).

An epidemiological study in Greenlandic Inuits suggested a key role for fish oil ( $\omega-3$ LCPUFA) in the prevention of atherosclerotic diseases (2). Following this landmark study, the health benefits of $\omega-3$ LCPUFAs, which predominantly comprise eicosapentaenoic acid (EPA; 22:5 n-3) and docosahexaenoic acid (DHA; 22:6 n-3), as part of a fatty acid-rich diet have been extensively researched; large-scale epidemiological studies, clinical outcome trial, and meta-analyses have reported a significant reduction in relative cardiovascular risk in response to intake of $\omega-3$ PUFAs (3-7). Therefore, the balance between EPA or DHA and AA in the human body is likely to be important for regulating the production of mediators, thus affecting vascular function. Notably, the serum EPA to AA (EPA/AA) ratio has been reported to be a promising biomarker for the risk of cardiovascular disease, not only in the general population (8), but also in a post-hoc analysis of the results of a clinical trial (9).

The efficacy of statins for primary and secondary prevention of cardiovascular disease has been established (10), and low-density-lipoprotein cholesterol (LDL-C)-lowering therapy with statins has been used as first-line treatment. Despite the significant LDL-C-lowering effects of statins, substantial residual cardiovascular risk remains (11), and numerous 
risk factors, including low levels of high-density-lipoprotein cholesterol and high levels of triglycerides, have attracted attention. Notably, an increase in plasma AA concentrations, and a decrease in plasma $\omega-3$ fatty acid concentrations and/or plasma $\omega-3 / \mathrm{AA}$ ratio, has been observed in patients treated with statins (12-17); these findings may be associated with the residual risk following the initiation of statin treatment. These findings suggest that statins regulate the endogenous biosynthesis of LCPUFAs.

LCPUFAs are endogenously biosynthesized from $\omega-6$ and $\omega-3$ PUFA precursors by position-specific desaturation and carbon-chain elongation reactions (18). Endogenous synthesis of LCPUFA and the degree of unsaturation of the biological membranes depend largely on the actions of the fatty acid desaturases FADS1 ( $\Delta 5$-desaturase), FADS2 $(\Delta 6-/ \Delta 8-/ \Delta 4$-desaturase) and putative FADS3, as well as elongation of very long-chain fatty acids proteins (ELOVLs) (19). A recent genome-wide association study demonstrated that the genes mediating endogenous synthesis of LCPUFAs contribute to variability in the efficiency of LCPUFA synthesis, and that synthesis is likely controlled by the levels of FADS2 and FADS1, as well as by the elongases, depending on genotype and metabolic state (19).

Since Fads1, Fads 2 and ELOVL fatty acid elongase 5 (Elovl5) are known to be involved in endogenous biosynthesis of AA and EPA (19), the present study investigated the effects of atorvastatin on the expression of these genes and the regulatory mechanism via the mevalonate cascade. Mouse 3T3-L1 adipocytes were used in the present study, since these cells are known to have a functional FADS pathway (20).

\section{Materials and methods}

3T3-L1 cell culture. Mouse 3T3-L1 preadipocytes were purchased from Health Science Research Resources Bank (Osaka, Japan). The cells were cultured in basal medium, which consisted of Dulbecco's modified Eagle's medium (DMEM; Gibco; Thermo Fisher Scientific, Inc., Waltham, MA, USA) supplemented with $10 \%$ fetal bovine serum (FBS; Hyclone; GE Healthcare Life Sciences, Logan, UT, USA) at $37^{\circ} \mathrm{C}$ in an atmosphere containing $5 \% \mathrm{CO}_{2}$. For adipocyte differentiation, 3T3-L1 preadipocytes were precultured in basal medium until $\sim 100 \%$ confluent, and were then cultured in differentiation medium containing DMEM, 10\% FBS, $10 \mu \mathrm{g} / \mathrm{ml}$ insulin, $0.25 \mu \mathrm{M}$ dexamethasone and $500 \mu \mathrm{M}$ 3-isobutyl-1-methylxanthine (Sigma-Aldrich; Merck KGaA, Darmstadt, Germany). After $48 \mathrm{~h}$, cells were transferred in differentiation medium without 3-isobutyl-1-methylxanthine and dexamethasone and were cultured at $37^{\circ} \mathrm{C}$ for $48 \mathrm{~h}$. The differentiated cells were exposed to 10, 30 and $100 \mu \mathrm{M}$ atorvastatin (Wako Pure Chemical Industries, Ltd., Osaka, Japan) in DMEM at $37^{\circ} \mathrm{C}$ for $48 \mathrm{~h}$ in combination with various agents: $10 \mu \mathrm{M}$ to $10 \mathrm{mM}$ Mevalonolactone, $10 \mu \mathrm{M}$ geranylgeranyl pyrophosphate (GGPP), $10 \mu \mathrm{M}$ farnesyl pyrophosphate (FPP) and $10 \mu \mathrm{M}$ cholesterol (Sigma-Aldrich; Merck KGaA). To examine the involvement of Rho kinase signaling, the cells were also incubated at $37^{\circ} \mathrm{C}$ for $48 \mathrm{~h}$ with 50 or $100 \mu \mathrm{M}$ Y-27632 (Wako Pure Chemical Industries, Ltd.), which is a selective inhibitor of Rho-associated protein kinase. For all experiments, the vehicle group contained cells treated with $0.1 \%$ methanol in the basal medium; the control group contained cells treated with $30 \mu \mathrm{M}$ atorvastatin in the basal medium.

Cell viability. Cell viability was assessed $48 \mathrm{~h}$ following treatment with the various reagents, by measuring mitochondrial activity, which reduces WST- 8 to formazan, using the Cell Counting Kit-8 assay (Dojindo Molecular Technologies, Inc., Kumamoto, Japan), according to manufacturer's protocol.

Reverse transcription-quantitative polymerase chain reaction (RT-qPCR). Total cellular RNA was extracted from 3T3-L1 cells using the RNeasy kit (Qiagen S.A.S., Courtaboeuf, France). The concentration of each sample was determined spectrophotometrically by measuring absorbance at $260 \mathrm{~nm}$. Reverse transcription was performed using the PrimeScript ${ }^{\circledR}$ RT reagent kit (Takara Bio, Inc., Otsu, Japan). The temperature protocol used was as follows: Reverse transcription at $37^{\circ} \mathrm{C}$ for $15 \mathrm{~min}$, inactivation of reverse transcriptase with heat treatment at $85^{\circ} \mathrm{C}$ for $5 \mathrm{sec}$, and storage at $4^{\circ} \mathrm{C}$. Quantification of mRNA expression was performed using SYBR ${ }^{\circledR}$ Premix Ex Taq ${ }^{\mathrm{TM}}$ II (Takara Bio, Inc.) on a Mx3005P qPCR system (Agilent Technologies Japan, Ltd., Tokyo, Japan). Thermocycling conditions were as follows: Enzyme activation $95^{\circ} \mathrm{C}$ for $30 \mathrm{sec}$, initial denaturation at $95^{\circ} \mathrm{C}$ for $5 \mathrm{sec}$, followed by 40 cycles of annealing/extension at $60^{\circ} \mathrm{C}$ for $30 \mathrm{sec}$. This was followed by a melt curve analysis according to the manufacturer's protocol to ensure specific amplification. The following primers were used: Mouse Fads1 sense, 5'-CCAGCTTTGAACCCACCA AGA-3' and antisense, 5'-CAGCAGGATGTGAAGCAGGTA GAC-3'; mouse Fads2 sense, 5'-GTCATCGACCGCAAG GTCTACA-3' and antisense, 5'-AGAACTTGCCCACGAAGT CCA-3'; mouse Elovl5 sense, 5'-CAGGGCCCAGAGCTTGTT AGTTTA-3' and antisense, 5'-ACCAAGGGTGCCATCAGA TTTC-3'; and mouse GAPDH sense, 5'-TGTGTCCGTCGT GGATCTGA-3' and antisense, 5'-TTGCTGTTGAAGTCG CAGGAG-3'. GAPDH expression was used as an internal standard. Relative mRNA expression was calculated using the comparative $\mathrm{Cq}$ method and was normalized to GAPDH expression (21).

Statistical analysis. All data are presented as the mean \pm standard deviation. SPSS software (version 11.5; SPSS, Inc., Chicago, IL, USA) was used to statistically analyze the data. Treatment effects were evaluated using one-way analysis of variance followed by Bonferroni multiple comparison test. $\mathrm{P}<0.05$ was considered to indicate a statistically significant difference.

\section{Results}

Effects of atorvastatin on cell viability of 3T3-L1 adipocytes. After $48 \mathrm{~h}$, atorvastatin inhibited cell viability in a dose-dependent manner. Atorvastatin significantly decreased cell viability to $84.5 \pm 1.9 \%$ at $30 \mu \mathrm{M}$ and to $82.0 \pm 8.1 \%$ at $100 \mu \mathrm{M}$ (Fig. 1A); these results are consistent with those of a previous study (22). Atorvastatin-mediated decreases in cell viability were reversed by the addition of mevalonolactone in a dose-dependent manner, with full reversal observed at $1 \mathrm{mM}$ (Fig. 1B); however, mevalonate in the absence of atorvastatin did not affect cell viability (Fig. 1B and C). 

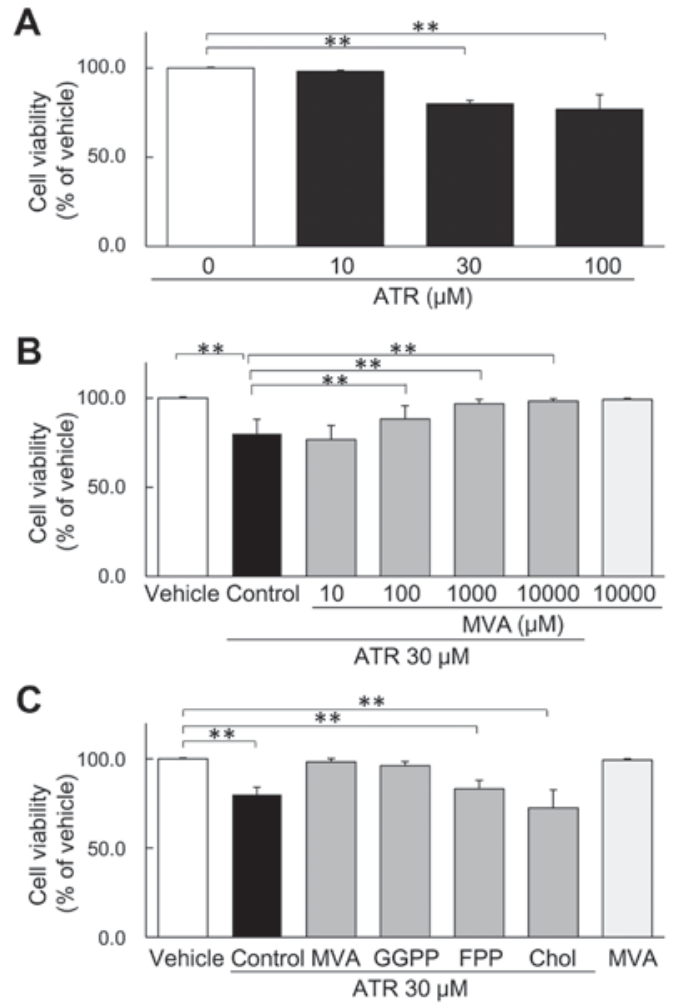

Figure 1. Cell viability of 3T3-L1 adipocytes. (A) Effects of ATR on cell viability. (B) Effects of MVA on viability of cells treated with ATR $(30 \mu \mathrm{M})$. (C) Effects of MVA $(1 \mathrm{mM})$, GGPP $(10 \mu \mathrm{M})$, FPP $(10 \mu \mathrm{M})$ and Chol $(10 \mu \mathrm{M})$ on viability of cells treated with ATR. Data are presented as the mean \pm standard deviation of five experiments. ${ }^{* *} \mathrm{P}<0.01$; one-way analysis of variance followed by Bonferroni multiple comparison test. ATR, atorvastatin; Chol, cholesterol; FPP, farnesyl pyrophosphate; GGPP, geranylgeranyl pyrophosphate; MVA, mevalonolactone.

These data suggested that the observed decrease in cell viability induced by atorvastatin may be due to a decrease in mevalonate or its metabolites occurring as a consequence of 3-hydroxy-3-methyl-glutaryl-coenzyme A (HMG-CoA) reductase inhibition. When metabolites of the mevalonate cascade were examined, GGPP at $10 \mu \mathrm{M}$ completely reversed atorvastatin-mediated decreases in cell viability, in a similar manner to mevalonolactone; however, FPP and cholesterol $(10 \mu \mathrm{M})$ had no such effect after $48 \mathrm{~h}$ (Fig. 1C).

Effects of atorvastatin on Fads1, Fads 2 and Elovl5 gene expression in 3T3-L1 adipocytes. The role of the intrinsic mevalonate cascade in Fads1, Fads2 and Elovl5 mRNA expression was investigated in mouse 3T3-L1 cells. Treatment with atorvastatin for $48 \mathrm{~h}$ increased the mRNA expression levels of Fadsl, Fads2 and Elovl5 to $105.4 \pm 3.5,109.5 \pm 7.9$ and $106.0 \pm 4.1 \%$ at $10 \mu \mathrm{M}$, and $168.1 \pm 1.5,235.6 \pm 5.5$ and $147.1 \pm 1.0 \%$ at $30 \mu \mathrm{M}$, respectively, in a dose-dependent manner (Fig. 2). Upregulation of these genes by atorvastatin $(30 \mu \mathrm{M})$ was reversed following the addition of mevalonolactone in a dose-dependent manner, with full reversal observed at $1 \mathrm{mM}$ (Fig. 3); however, mevalonate in the absence of atorvastatin did not significantly affect Fads1, Fads2 and Elovl5 mRNA expression (Figs. 3 and 4). In addition, the present study examined the roles of mevalonate metabolites on the mRNA expression levels of Fads1, Fads2 and Elovl5 in atorvastatin-treated cells; treatment with $1 \mathrm{mM}$
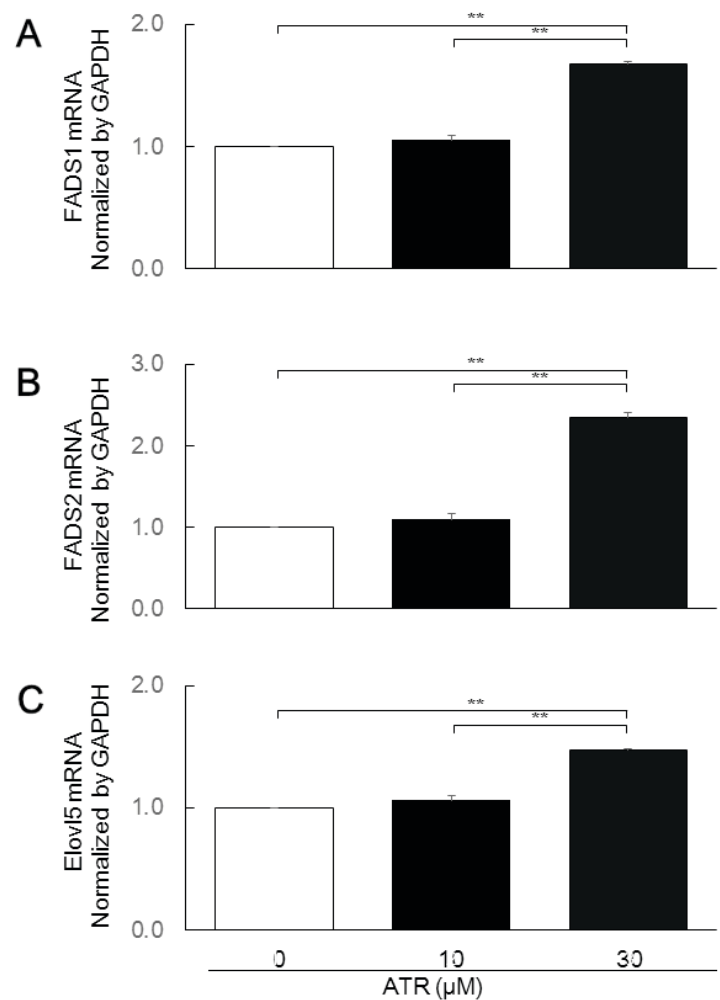

Figure 2. Effects of ATR on (A) Fads1, (B) Fads2 and (C) Elovl5 mRNA expression in 3T3-L1 adipocytes. Data are presented as the mean \pm standard deviation of three experiments. ${ }^{* *} \mathrm{P}<0.01$; one-way analysis of variance followed by Bonferroni multiple comparison test. ATR, atorvastatin; Elovl5, ELOVL fatty acid elongase 5; Fads, fatty acid desaturase.
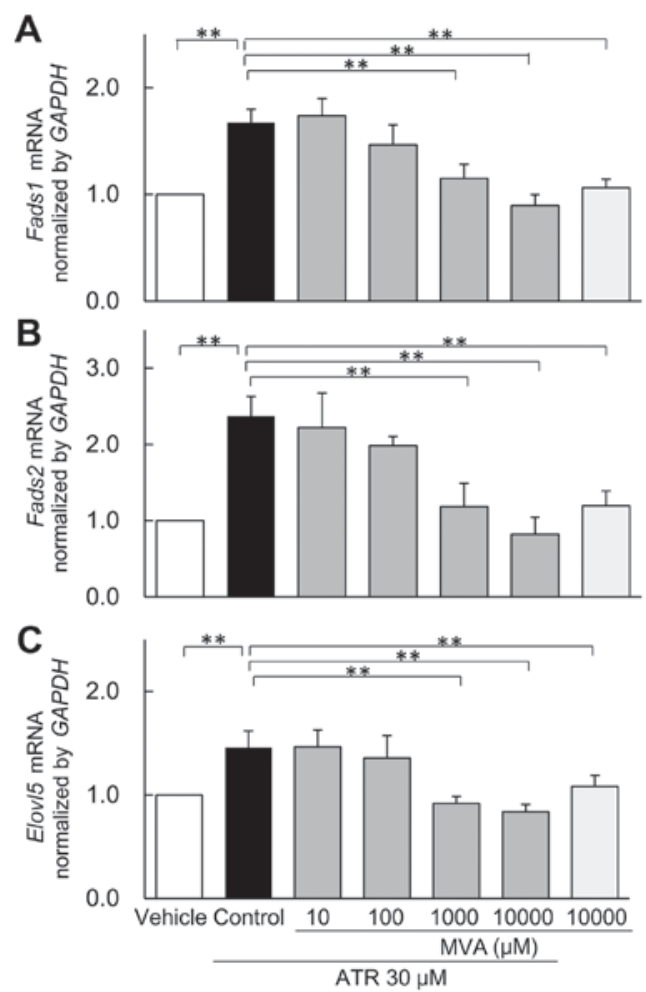

Figure 3. Effects of ATR and MVA on (A) Fads1, (B) Fads2 and (C) Elovl5 mRNA expression in 3T3-L1 adipocytes. Data are presented as the mean \pm standard deviation of three experiments. ${ }^{* *} \mathrm{P}<0.01$; one-way analysis of variance followed by Bonferroni multiple comparison test. ATR, atorvastatin; Elovl5, ELOVL fatty acid elongase 5; Fads, fatty acid desaturase; MVA, mevalonolactone. 

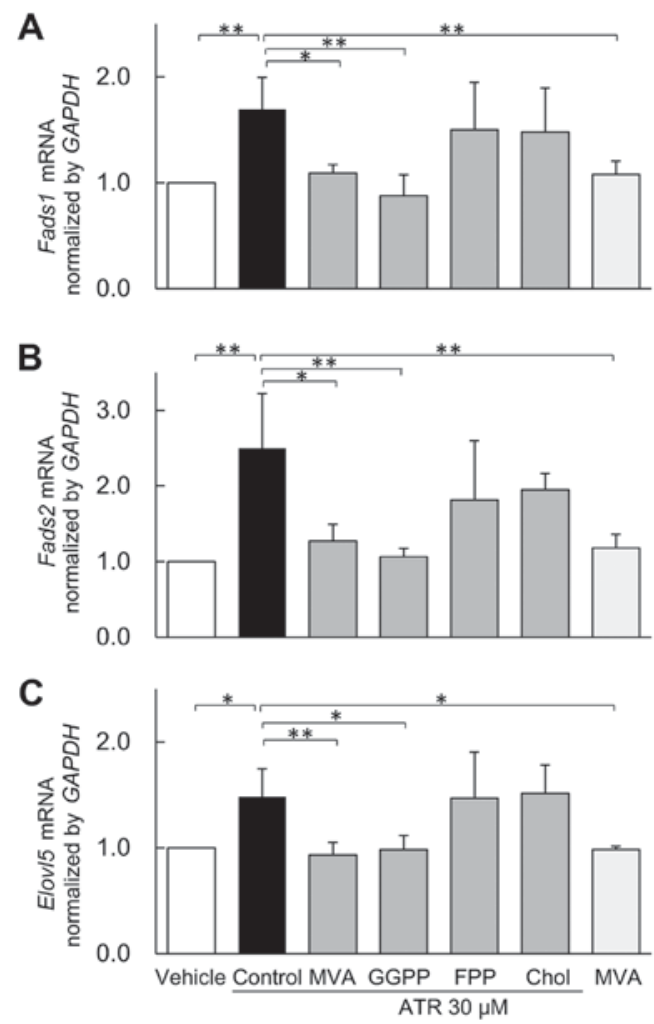

Figure 4. Effects of MVA (1 mM), GGPP $(10 \mu \mathrm{M})$, FPP $(10 \mu \mathrm{M})$ and Chol $(10 \mu \mathrm{M})$ on (A) Fads1, (B) Fads2 and (C) Elovl5 expression in 3T3-L1 adipocytes treated with ATR. Data are presented as the mean \pm standard deviation of three experiments. ${ }^{*} \mathrm{P}<0.05,{ }^{* *} \mathrm{P}<0.01$; one-way analysis of variance followed by Bonferroni multiple comparison test. ATR, atorvastatin; Chol, cholesterol; Elovl5, ELOVL fatty acid elongase 5; Fads, fatty acid desaturase; FPP, farnesyl pyrophosphate; GGPP, geranylgeranyl pyrophosphate; MVA, mevalonolactone.

mevalonolactone or $10 \mu \mathrm{M} \mathrm{GGPP} \mathrm{reversed} \mathrm{atorvastatin-induced}$ upregulated mRNA expression, whereas FPP and cholesterol did not (Fig. 4). These data suggested that the observed increases in Fads1, Fads2 and Elovl5 mRNA expression induced by atorvastatin may be due to decreases in mevalonate metabolites, particularly GGPP, occurring as a consequence of atorvastatin-induced $\mathrm{HMG}-\mathrm{CoA}$ reductase inhibition.

Effects of the Rho kinase inhibitor Y-27632 on Fads1, Fads2 and Elovl5 gene expression. Since GGPP is responsible for post-translational activation of the small GTPase RhoA, it is possible that the atorvastatin-induced depletion of GGPP leads to inhibition of RhoA and its effector Rho-associated protein kinase (ROCK), which is one of the major downstream targets of RhoA. Therefore, the present study examined whether Y-27632, a Rho kinase inhibitor, may abolish the effects of MVA and GGPP to restore the atorvastatin-induced upregulation of Fadsl, Fads2 and Elovl5 mRNA expression. Y-27632 dose-dependently inhibited the both MVA and GGPP-induced suppression of Fadsl, Fads2 and Elovl5 mRNA expression in atorvastatin-treated 3T3-L1 adipocytes (Fig. 5).

\section{Discussion}

The main objective of the present study was to verify whether treatment with atorvastatin, an HMG-CoA reductase-specific
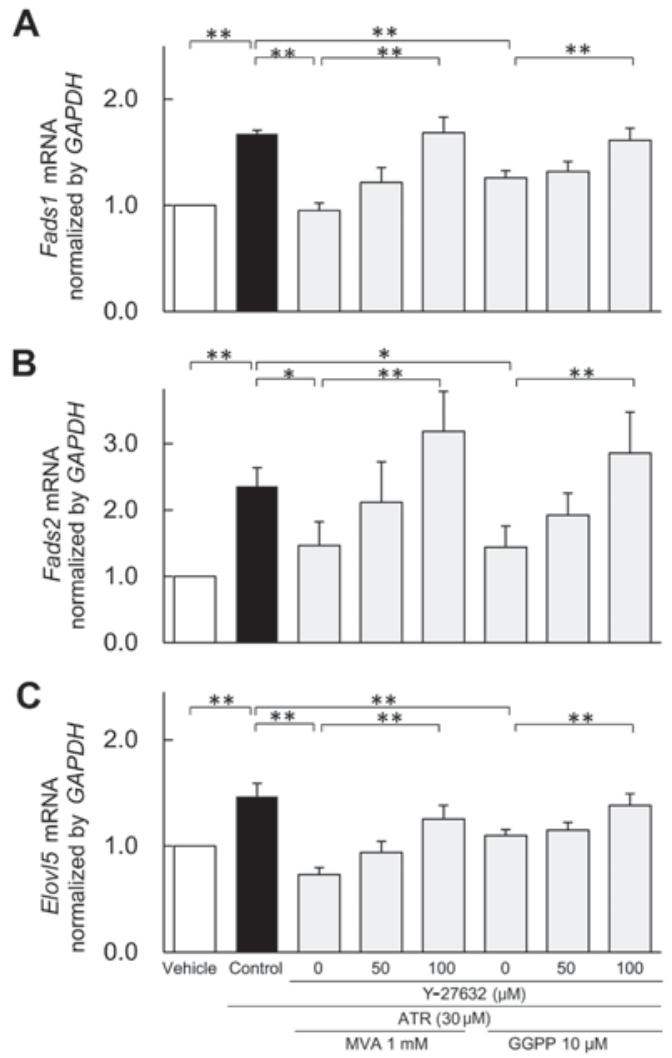

Figure 5. Effects of the Rho kinase inhibitor Y-27632 on (A) Fads1, (B) Fads2 and (C) Elovl5 mRNA expression in 3T3-L1 adipocytes treated with ATR and GGPP or mevalonate. Data are presented as the mean \pm standard deviation of three experiments. ${ }^{*} \mathrm{P}<0.05,{ }^{* *} \mathrm{P}<0.01$; one-way analysis of variance followe-d by Bonferroni multiple comparison test. ATR, atorvastatin; Elovl5, ELOVL fatty acid elongase 5; Fads, fatty acid desaturase; GGPP, geranylgeranyl pyrophosphate; MVA, mevalonolactone.

inhibitor (22), affects the mRNA expression levels of Fads1, Fads2 and Elovl5, and to elucidate the mechanisms involved. The present study demonstrated that although atorvastatin decreased cell viability, as reported previously (22), the drug upregulates the mRNA expression levels of Fadsl, Fads2 and Elovl5 in a dose-dependent manner in 3T3-L1 adipocytes. Furthermore, the findings of the present study suggested that cellular depletion of mevalonate and GGPP may be involved in the upregulation of Fads1, Fads2 and Elovl5 expression, and the decrease in cell viability induced by atorvastatin. By inhibiting HMG-CoA reductase, which is a rate-limiting enzyme in the cholesterol synthesis pathway, atorvastatin may decrease the levels of these compounds, which are intermediates of the pathway. Since GGPP is responsible for the post-translational activation of RhoA, atorvastatin-induced depletion of GGPP may lead to inhibition of RhoA and its effector ROCK. Notably, the present study revealed that treatment with a ROCK inhibitor (Y-27632) reversed the GGPP-induced suppression of Fads1, Fads 2 and Elovl5 expression in atorvastatin-treated 3T3-L1 adipocytes. To the best of our knowledge, the present study is the first to report the involvement of GGPP-dependent Rho and ROCK in atorvastatin-induced upregulation of Fadsl, Fads2 and Elovl5 mRNA expression.

The effect of statins on the endogenous synthesis of LCPUFAs was first reported by Hrboticky et al (23). Using human monocytic Mono-Mac-6 and HepG2 hepatoma cells, 
Cell Fads2 upregulation has been detected in human lymphoblasts (30), the present study is the first, to the best of our knowledge, to report the statin-induced upregulation of Elovl5 mRNA expression.

Notably, the present study indicated that the atorvastatin-induced decrease in cell viability and upregulation of Fads1, Fads 2 and Elovl5 expression were fully reversed by co-incubation with mevalonolactone and its metabolite GGPP, but not by cholesterol. Although statin-induced suppression of cell proliferation and viability, and statin-induced apoptosis, have been reported to be dependent on mevalonic acid and GGPP in various cell types $(22,31,32)$, the present study is the first, to the best of our knowledge, to report that atorvastatin-induced upregulation of Fads1, Fads 2 and Elovl5 expression is reversed by the addition of GGPP. In addition, the present study revealed that the ROCK inhibitor Y-27632 may abolish the GGPP-induced reversal of atorvastatin-mediated upregulation of these genes.

The findings of the present study demonstrated that statins may upregulate these genes via the GGPP-dependent ROCK pathway in mouse 3T3-L1 cells (Fig. 5). A similar finding was reported in a previous study regarding fibulin-2 expression in human coronary artery smooth muscle cells (33), in which simvastatin increased the mRNA expression levels of the extracellular matrix protein fibulin-2 through RhoA and the Rho kinase-mediated pathway. RhoA/ROCK signaling pathways serve central roles in regulating cell adhesion, migration, motility, contraction, apoptosis and proliferation $(34,35)$. The RhoA/ROCK signaling pathway is also capable of controlling gene and protein expression (36,37); however, the mechanism by which RhoA/ROCK affects Fads1, Fads 2 and Elovl5 expression remains unknown.

Notably, increases in plasma AA concentration, and decreases in plasma $\omega-3$ LCPUFA concentration and/or $\omega$-3/AA ratio, have been observed in patients treated with statins $(12-16,26,27)$. Although atorvastatin upregulates Fads1, Fads 2 and Elovl5 mRNA expression to increase the endogenous synthesis of LCPUFAs (Fig. 6), it remains unclear from the present study why statin treatment predominantly increases $\omega-6$ (AA), but reduces $\omega$-3 (EPA and DHA) LCPUFAs in patients. There are several possibilities, as follows: The substrates for synthesis of $\omega-6$ LCPUFAs, such as linoleic acid (LA; 18:2 n-6), are present in much larger quantities than those for the synthesis of $\omega-3$ LCPUFAs, such as $\alpha$-linolenic acid (ALA, 18:3 n-3), in in vivo and in vitro systems; therefore, conversion to AA may be dominant. This possibility is supported by the findings of a previous study, which suggested that ALA supplementation may reduce lovastatin-induced elevation of AA and increase cellular lipoprotein EPA and DHA levels in HepG2 cells (38). Another possibility is that synthesis of $\omega-6$ and $\omega-3$ LCPUFAs differs dependent on cell type. In a previous study, LA, ALA and stearic acid were reported to be metabolized differently in THP-1 and HepG2 cells, and their increased conversion by simvastatin was lower in HepG2 cells compared with in THP-1 cells; however, the precise underlying mechanism remains unknown (24).

The AA-dominant endogenous synthesis of LCPUFAs, which results in decreased plasma $\omega-3$ concentration and/or $\omega-3 / \mathrm{AA}$ ratio, during statin treatment may be clinically important, since serum EPA/AA ratio has been reported as a 
promising biomarker for the risk of cardiovascular disease, not only in the general population (8), but also in a post-hoc analysis of the results of a clinical trial (9). Therefore, it seems reasonable to recommend $\omega-3$ LCPUFAs supplementation for patients on statin treatment, in order to maintain plasma $\omega-3$ concentrations and the $\omega-3 / \mathrm{AA}$ ratio. Furthermore, a clinical study demonstrated the effectiveness of EPA in reducing cardiovascular events in Japanese patients treated with statins (4).

The present study has some limitations. Mouse 3T3-L1 adipocytes were used as a model to investigate the endogenous synthesis of LCPUFAs, since 3T3-L1 adipocytes have been reported to possess a functional FADS pathway (20); however, it is well known that LCPUFAs in the plasma are largely produced by the liver, and hepatic regulation of endogenous LCPUFA synthesis may be critical. Nevertheless, in our preliminary study using hepatocytes, similar results to those using 3T3-L1 adipocytes were obtained (unpublished data). In addition, it is necessary to demonstrate statin-induced upregulation of Fads1, Fads 2 and Elovl5 gene expression via the GGPP-dependent Rho kinase pathway in an in vivo model.

In conclusion, the present study demonstrated that atorvastatin may affect the endogenous synthesis of LCPUFAs by regulating Fads1, Fads 2 and Elovl5 expression via the GGPP-dependent Rho kinase pathway in mouse 3T3-L1 cells.

\section{References}

1. Weksler BB: Prostanoids and NSAIDs in cardiovascular biology and disease. Curr Atheroscler Rep 17: 41, 2015.

2. Bang HO, Dyerberg $J$ and Nielsen AB: Plasma lipid and lipoprotein pattern in Greenlandic West-coast Eskimos. Lancet 1: 1143-1145, 1971.

3. Tavazzi L, Maggioni AP, Marchioli R, Barlera S, Franzosi MG, Latini R, Lucci D, Nicolosi GL, Porcu M and Tognoni G; Gissi-HF Investigators: Effect of $n-3$ polyunsaturated fatty acids in patients with chronic heart failure (the GISSI-HF trial): A randomised, double-blind, placebo-controlled trial. Lancet 372 : 1223-1230, 2008.

4. Yokoyama M, Origasa H, Matsuzaki M, Matsuzawa Y, Saito Y, Ishikawa Y, Oikawa S, Sasaki J, Hishida H, Itakura $\mathrm{H}$, et al: Effects of eicosapentaenoic acid on major coronary events in hypercholesterolaemic patients (JELIS): A randomised open-label, blinded endpoint analysis. Lancet 369: 1090-1098, 2007.

5. Dietary supplementation with n-3 polyunsaturated fatty acids and vitamin $\mathrm{E}$ after myocardial infarction: Results of the GISSI-Prevenzione trial. Gruppo Italiano per lo Studio della Sopravvivenza nell'Infarto miocardico. Lancet 354: 447-455, 1999.

6. Morris MC, Manson JE, Rosner B, Buring JE, Willett WC and Hennekens $\mathrm{CH}$ : Fish consumption and cardiovascular disease in the physicians' health study: A prospective study. Am J Epidemiol 142: 166-175, 1995.

7. Burr ML, Fehily AM, Gilbert JF, Rogers S, Holliday RM, Sweetnam PM, Elwood PC and Deadman NM: Effects of changes in fat, fish, and fibre intakes on death and myocardial reinfarction: Diet and reinfarction trial (DART). Lancet 2: 757-761, 1989.

8. Ninomiya T, Nagata M, Hata J, Hirakawa Y, Ozawa M, Yoshida D, Ohara T, Kishimoto H, Mukai N, Fukuhara M, et al: Association between ratio of serum eicosapentaenoic acid to arachidonic acid and risk of cardiovascular disease: The Hisayama Study. Atherosclerosis 231: 261-267, 2013.

9. Itakura H, Yokoyama M, Matsuzaki M, Saito Y, Origasa H, Ishikawa Y, Oikawa S, Sasaki J, Hishida H, Kita T, et al: Relationships between plasma fatty acid composition and coronary artery disease. J Atheroscler Thromb 18: 99-107, 2011.
10. Cholesterol Treatment Trialists' (CTT) Collaboration, Fulcher J, O'Connell R, Voysey M, Emberson J, Blackwell L, Mihaylova B, Simes J, Collins R, Kirby A, et al: Efficacy and safety of LDL-lowering therapy among men and women: Meta-analysis of individual data from 174,000 participants in 27 randomised trials. Lancet 385: 1397-1405, 2015.

11. Sampson UK, Fazio S and Linton MF: Residual cardiovascular risk despite optimal LDL cholesterol reduction with statins: The evidence, etiology, and therapeutic challenges. Curr Atheroscler Rep 14: 1-10, 2012.

12. Nozue T, Yamamoto S, Tohyama S, Fukui K, Umezawa S, Onishi Y, Kunishima T, Sato A, Nozato T, Miyake S, et al: Comparison of effects of serum n-3 to n-6 polyunsaturated fatty acid ratios on coronary atherosclerosis in patients treated with pitavastatin or pravastatin undergoing percutaneous coronary intervention. Am J Cardiol 111: 1570-1575, 2013

13. Kurisu S, Ishibashi K, Kato Y, Mitsuba N, Dohi Y, Nishioka K and Kihara Y: Effects of lipid-lowering therapy with strong statin on serum polyunsaturated fatty acid levels in patients with coronary artery disease. Heart Vessels 28: 34-38, 2013.

14. Jula A, Marniemi J, Rönnemaa T, Virtanen A and Huupponen R: Effects of diet and simvastatin on fatty acid composition in hypercholesterolemic men: A randomized controlled trial. Arterioscler Thromb Vasc Biol 25: 1952-1959, 2005.

15. de Lorgeril M, Salen P, Guiraud A, Zeghichi S, Boucher F and de Leiris J: Lipid-lowering drugs and essential omega-6 and omega-3 fatty acids in patients with coronary heart disease. Nutr Metab Cardiovasc Dis 15: 36-41, 2005.

16. Harris JI, Hibbeln JR, Mackey RH and Muldoon MF: Statin treatment alters serum n-3 and n- 6 fatty acids in hypercholesterolemic patients. Prostaglandins Leukot Essent Fatty Acids 71: 263-269, 2004

17. Nakamura N, Hamazaki T, Ohta M, Okuda K, Urakaze M, Sawazaki S, Yamazaki K, Satoh A, Temaru R, Ishikura Y, et al: Joint effects of HMG-CoA reductase inhibitors and eicosapentaenoic acids on serum lipid profile and plasma fatty acid concentrations in patients with hyperlipidemia. Int J Clin Lab Res 29: 22-25, 1999.

18. Abedi E and Sahari MA: Long-chain polyunsaturated fatty acid sources and evaluation of their nutritional and functional properties. Food Sci Nutr 2: 443-463, 2014.

19. Zhang JY, Kothapalli KS and Brenna JT: Desaturase and elongase-limiting endogenous long-chain polyunsaturated fatty acid biosynthesis. Curr Opin Clin Nutr Metab Care 19: 103-110, 2016.

20. Ralston JC, Matravadia S, Gaudio N, Holloway GP and Mutch DM: Polyunsaturated fatty acid regulation of adipocyte FADS1 and FADS2 expression and function. Obesity (Silver Spring) 23: 725-728, 2015.

21. Livak KJ and Schmittgen TD: Analysis of relative gene expression data using real-time quantitative PCR and the 2(-Delta Delta C(T)) method. Methods 25: 402-408, 2001.

22. Siddals KW, Marshman E, Westwood M and Gibson JM: Abrogation of insulin-like growth factor-I (IGF-I) and insulin action by mevalonic acid depletion: Synergy between protein prenylation and receptor glycosylation pathways. J Biol Chem 279: 38353-38359, 2004

23. Hrboticky N, Tang L, Zimmer B, Lux I and Weber PC: Lovastatin increases arachidonic acid levels and stimulates thromboxane synthesis in human liver and monocytic cell lines. J Clin Invest 93: 195-203, 1994.

24. Risé P, Ghezzi S, Priori I and Galli C: Differential modulation by simvastatin of the metabolic pathways in the n-9, n- 6 and n-3 fatty acid series, in human monocytic and hepatocytic cell lines. Biochem Pharmacol 69: 1095-1100, 2005.

25. Dwight JF, Mendes Ribeiro AC and Hendry BM: Effects of HMG-CoA reductase inhibition on erythrocyte membrane cholesterol and acyl chain composition. Clin Chim Acta 256: 53-63, 1996.

26. Nakamura N, Hamazaki T, Jokaji H, Minami S and Kobayashi M: Effect of HMG-CoA reductase inhibitors on plasma polyunsaturated fatty acid concentrations in patients with hyperlipidemia. Int J Clin Lab Res 28: 192-195, 1998.

27. Nozue T and Michishita I: Statin treatment alters serum n-3 to n-6 polyunsaturated fatty acids ratio in patients with dyslipidemia. Lipids Health Dis 14: 67, 2015.

28. Lohner S, Fekete K, Marosvolgyi T and Decsi T: Gender differences in the long-chain polyunsaturated fatty acid status: Systematic review of 51 publications. Ann Nutr Metab 62: 98-112, 2013 
29. Sibbons CM, Brenna JT, Lawrence P, Hoile SP, Clarke-Harris R, Lillycrop KA and Burdge GC: Effect of sex hormones on n-3 polyunsaturated fatty acid biosynthesis in HepG2 cells and in human primary hepatocytes. Prostaglandins Leukot Essent Fatty Acids 90: 47-54, 2014

30. Reardon HT, Zhang J, Kothapalli KS, Kim AJ, Park WJ and Brenna JT: Insertion-deletions in a FADS2 intron 1 conserved regulatory locus control expression of fatty acid desaturases 1 and 2 and modulate response to simvastatin. Prostaglandins Leukot Essent Fatty Acids 87: 25-33, 2012.

31. Tatsuno I, Tanaka T, Oeda T, Yasuda T, Kitagawa M, Saito Y and Hirai A: Geranylgeranylpyrophosphate, a metabolite of mevalonate, regulates the cell cycle progression and DNA synthesis in human lymphocytes. Biochem Biophys Res Commun 241: 376-382, 1997.

32. Hirai A, Nakamura S, Noguchi Y, Yasuda T, Kitagawa M, Tatsuno I, Oeda T, Tahara K, Terano T, Narumiya S, et al: Geranylgeranylated rho small GTPase(s) are essential for the degradation of $\mathrm{p} 27^{\mathrm{Kipl}}$ and facilitate the progression from $\mathrm{G} 1$ to $\mathrm{S}$ phase in growth-stimulated rat FRTL-5 cells. J Biol Chem 272: 13-16, 1997.
33. Serra N, Rosales R, Masana L and Vallvé JC: Simvastatin increases fibulin-2 expression in human coronary artery smooth muscle cells via RhoA/Rho-kinase signaling pathway inhibition. PLoS One 10: e0133875, 2015.

34. Etienne-Manneville S and Hall A: Rho GTPases in cell biology. Nature 420: 629-635, 2002

35. Takai Y, Sasaki T, Tanaka K and Nakanishi H: Rho as a regulator of the cytoskeleton. Trends Biochem Sci 20: 227-231, 1995.

36. Cai A, Li L and Zhou Y: Pathophysiological effects of RhoA and Rho-associated kinase on cardiovascular system. J Hypertens 34: 3-10, 2016.

37. O'Cochlain DF, Perez-Terzic C, Reyes S, Kane GC, Behfar A, Hodgson DM, Strommen JA, Liu XK, van den Broek W, Wansink DG, et al: Transgenic overexpression of human DMPK accumulates into hypertrophic cardiomyopathy, myotonic myopathy and hypotension traits of myotonic dystrophy. Hum Mol Genet 13: 2505-2518, 2004.

38. Hrboticky N, Zimmer B and Weber PC: $\alpha$-Linoleic acid reduces the lovastatin-induced rise in arachidonic acid and elevates cellular and lipoprotein eicosapentaenoic acid and docosahexaenoic acid levels in Hep G2 cells. J Nutr Biochem 7: 452-471, 1996. 